\title{
Mining Topic-level Opinion Influence in Microblog
}

\author{
Daifeng Li \\ Dept. of Computer Science \\ and Technology \\ Tsinghua University \\ Beijing, China \\ Idf3824@yahoo.com.cn \\ Jie Tang \\ Dept. of Computer Science \\ and Technology \\ Tsinghua University \\ Beijing, China \\ jery.tang@gmail.com
}

\author{
Xin Shuai \\ School of Informatics and \\ Computing \\ Indiana University \\ Bloomington \\ IN, USA \\ xshuai@indiana.edu \\ Ying Ding \\ School of Library and \\ Information Science \\ Indiana University \\ Bloomington \\ IN, USA \\ dingying@indiana.edu
}

\begin{abstract}
This paper proposes a Topic-Level Opinion Influence Model (TOIM) that simultaneously incorporates topic factor and social influence in a two-stage probabilistic framework. Users' historical messages and social interaction records are leveraged by TOIM to construct their historical opinions and neighbors' opinion influence through a statistical learning process, which can be further utilized to predict users' future opinions on some specific topic. We evaluate our TOIM on a large-scaled dataset from Tencent Weibo, one of the largest microblogging website in China. The experimental results show that TIOM can better predict users' opinion than other baseline methods.
\end{abstract}

\section{Categories and Subject Descriptors}

H.2.8 [Database and Management]: Data Mining; J.4 [Computer Applications]: Social and Behavioral Science

\section{Keywords}

Opinion Mining, Sentiment Analysis, Social Influence, Topic Modeling

\section{INTRODUCTION}

Opinion mining, or sentiment analysis, aims to classify polarity of a document into positive or negative. There're two important factors that should be taken into considerations. One, opinions and topics are closely related. The online discussions around some entity, or object, often cover a mixture of features/topics related to that entity with dif-

Permission to make digital or hard copies of all or part of this work for personal or classroom use is granted without fee provided that copies are not made or distributed for profit or commercial advantage and that copies bear this notice and the full citation on the first page. To copy otherwise, to republish, to post on servers or to redistribute to lists, requires prior specific permission and/or a fee.

CIKM'12, October 29-November 2, 2012, Maui, HI, USA

Copyright 2012 ACM 978-1-4503-1156-4/12/10 ...\$10.00. ferent preferentials. Different opinions may be expressed by users towards different topics, where users may like some aspects of an entity but dislike other aspects. Two, users' opinions are subject to social influence. The rise of social media puts the sentiment analysis in the context of social network. Users not only express their individual opinions, but also exchange opinions with others. In the context of opinion mining, social influence refers to the phenomenon that one is inclined to agree (positive influence) or disagree (negative influence) with his/her neighbors' opinions with different degrees, depending on the influence strengths.

Several opinion mining related studies are in line with our work. Mei et. al [8] proposed Topic-Sentiment Mixture (TSM) model that can reveal latent topical facets in a Weblog collections, and their associated sentiments. Lin et. al [5] proposed a joint sentiment/topic (JST) model based on LDA that can detect topic and sentiment simultaneously. Both TSM and JST tried to model topic and sentiment at the same time but social influence is not considered. Our paper tries to incorporate topic modeling, social influence and sentiment analysis into a two-stages model(stage 1 is to build topic level influential relationship among users, while stage 2 is to calculate the strength of opinion influence based on stage 1) to classify users' polarities.

We show a typical scenario of topic level opinion influence analysis on Tencent Microblog (a chinese microblogging website) in Figure 1. Like Twitter, Weibo users can post messages of up to 140 chinese characters and follow other users to read their messages. Followers can reply to other users' message by leaving their own comment, whose opinions can be mined from the comments. Two types of entities (user and message) and multiple types of relations (user posts/comments on message, user replies to another user) constitute a heterogenous network built on Tencent Weibo. Specifically, Lucy comments on both Lee and Peggy's messages and replies to both of them on the visual effect aspect of the movie Titanic 3D. Given the topological and text information, we aim to generate a topic opinion influence ego-network, with Lucy at the center influenced by Lee and Peggy. Their historical opinion distributions over positive/negative opinion, as well as type (agree means pos- 


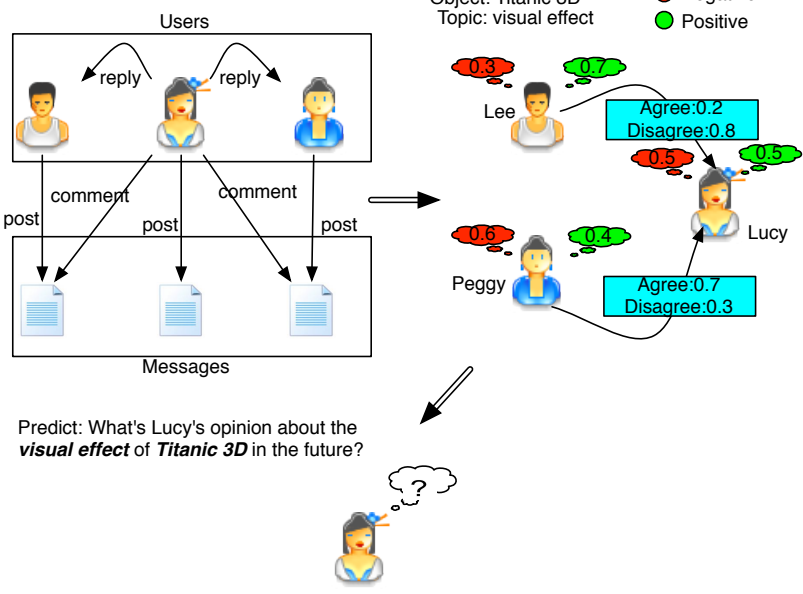

Figure 1: Motivating example

itive influence while disagree means negative influence) and strength (agree/disagree probability) of the opinion influence between Lucy and Lee/Peggy are calculated. Finally, how can we predict Lucy's further opinion by jointly considering her own opinion preference and opinion influence from Lee and Peggy?

To solve the problem in Figure 1, we propose a Topic-level Opinion Influence Model (TOIM) that simultaneously incorporates topic factor and social influence in a unified probabilistic framework; users' historical messages and social interaction records are leveraged by TOIM to construct their historical opinions and neighbors' opinion influence through a statistical learning process, which can be further utilized to predict users' future opinions towards some specific topic. Our main conclusions include:

- we propose a probabilistic model to capture the dual effect of topic preference and social influence on opinion prediction problem.

- we testify our model on a new and large-scaled chinese microblog dataset from Tencent Weibo.

This paper is organized as follows: Section 2 defines the problem. Section 3 explains the mechanism of TOIM and illustrates the process of model learning process. Section 4 shows the experimental results and case studies. Section 5 lists related literature and Section 6 concludes the study.

\section{PROBLEM DEFINITION}

In this section, we formally define our problem of predicting a user's opinion regarding certain topic by jointly considering the user's historical opinion and opinion influence from the neighbors. Our problem starts with a Heterogeneous Social Network on Tencent Weibo, where nodes include all users (i.e. followers and followees) and all messages (i.e. posts and comments), and edges include all actions from users to messages (i.e. post and comment) and all connections from users to users (i.e. reply). Specifically, given a query object, a sub-graph $G=(U, M, A, E)$ can be extracted from the Heterogeneous Social Network where $U=\left\{u_{i}\right\}_{i=1}^{V}$ is a set of users once posted or commented on messages about the object, $M=\left\{m_{i}\right\}_{i=1}^{D}$ is a set of messages posted or commented from $u_{i} \in U$, $A=\left\{\left(u_{i}, m_{i}\right) \mid u_{i} \in U, m_{i} \in M\right\}$ is a set of edges indicating $u_{i}$ posted or commented on $m_{j}$, and $E=\left\{\left(u_{i}, u_{j}\right) \mid u_{i}, u_{j} \in U\right\}$ is a set of edges indicating $u_{j}$ replied to $u_{i}$. Based on $G$, we list several formal definitions as follows:

DEFINITION 1. [Vocabulary] All distinct words from $M$ constitute a vocabulary $W=\left\{w_{i}\right\}_{i=1}^{X}$. According to the word property, we further define noun vocabulary $W_{N}=\left\{n_{i}\right\}_{i=1}^{N}$ where $n_{i}$ is a noun and opinion vocabulary $W_{O}=\left\{o w_{i}\right\}_{i=1}^{Q}$ where $o_{j}$ is an adjective or verb. The intuition is that a noun represents a topic while an adjective or verb indicates an opinion of the noun

DEFINITION 2. [Opinion Words] In a sentence, the opinion on a noun is often expressed by verbs or adjective. E.g. I like iphone4, Adele is a marvelous singer. Such words are called opinion words. We use $O\left(n_{i}\right)$ to denote the opinion word of a noun $n_{i}$ and $O\left(n_{i}\right) \in W_{O}$.

DEFINITION 3. [Topic-User-Opinion Distribution] Different users show different opinions towards the same topic. We define a topic-user-opinion distribution $\boldsymbol{\Psi}=$ $\left\{\psi_{i, j}^{k}\right\}$

$K \times V \times 2$ where $\psi_{i, j}^{k}$ denotes the probability that user $u_{i}$ prefers opinion $o_{j}$ given topic $t_{k}$ and $o_{j} \in\{-1,+1\}$.

DEFINITION 4. [Topic Opinion Neighbors] For user $u_{i}$, all users that $u_{i}$ replied to regarding to topic $t_{k}$ constitute a set $O N\left(u_{i}, t_{k}\right)$ which is called $u_{i}$ 's topic opinion neighbors around $t_{k}$. Each user $u_{j} \in O N\left(u_{i}, t_{k}\right)$ can influence $u_{i}$ 's opinion on $t_{k}$.

DEFINITION 5. [Topic-Opinion Influence] For any $u_{j} \in O N\left(u_{i}, t_{k}\right)$, the influence of $u_{j}$ on $u_{i}$ can be measured by $\boldsymbol{\Omega}=\left\{\omega_{i, j, \text { agree }}^{k}\right\}_{K \times V \times V \times 2} \cup\left\{\omega_{i, j, \text { disagree }}^{k}\right\}_{K \times V \times V \times 2}$ where $\omega_{i, j, \text { agree }}^{k}$ denotes the probability that $u_{i}$ agrees with

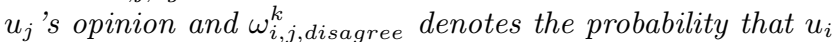
disagrees with $u_{j}$ 's opinion on topic $t_{k}$.

The most important four parameters are $\Theta, \Phi, \Psi$ and $\Omega$, which bind the user, topic, opinion and influence in a unified framework. Our task can be reduced to the following two steps:

- First, given $G$, how to estimate $\Theta, \Phi, \Psi$ and $\Omega$ ?

- Second, given user $u_{i}$ and topic $t_{j}$, if $\Theta, \Phi, \Psi$ and $\Omega$ are known, how to predict $u_{i}$ 's opinion of $t_{j}$ if $u_{i}$ post or comment on a new message?

\section{MODEL DESCRIPTION}

\subsection{Sentiment Analysis}

\subsubsection{Message-level sentiment}

Message level sentiment analysis captures the opinion word $O\left(n_{i}\right)$ for a noun $n_{i}$ and judge the polarity of $O\left(n_{i}\right)$ in the context of a message. First, a parse tree is constructed to exhibit the syntactic structure of a sentence and dependency relations between words. Consequently, $O\left(n_{i}\right)$ can be spotted by analyzing the structure of parse tree. Second, the polarity of $O\left(n_{i}\right)$ is judged by searching a corpus of Chinese sentimental words lexicon provided by Tsinghua NLP group, which consists of 5,567 positive and 4,479 negative words. Besides, two additional rules are applied to modify sentimental relation: whether there exists negation word, 
like not, don't, etc.; and whether there exists adversative relation between $n_{i}$ and $O\left(n_{i}\right)$, like but, however, etc.

Based on our experiment, the number of $n_{i}-O\left(n_{i}\right)$ pairs are usually small, due to the short and noisy feature of microblog messages. In order to overcome the limitation of data sparsity, we consider the statistical co-occrrence relations from all messages we collected. For each distinct noun $n_{i} \in W_{N}$ we find out all adjectives/verbs $o w_{i} \in W_{O}$ that co-occur with $n_{i}$ in all messages and pick out the top 20 most frequent co-occurrent $o w_{1}, \ldots, o w_{20}$, which constitutes a set $O S\left(n_{j}\right)$. For each $o w_{j} \in O S\left(n_{j}\right)$, we define a statistical dependence $(S D)$ :

$$
S D\left(n_{i}, o w_{j}\right)=\frac{C O\left(n_{i}, o w_{j}\right)}{A V E D I S\left(n_{i}, o w_{j}\right)}, j=1, \ldots, 20
$$

where $C O\left(n_{i}, o_{j}\right)$ denotes the total number of co-occurent frequency of $n_{i}$ and $o w_{j}$, and $A V E D I S\left(n_{i}, o w_{j}\right)$ denotes the average distance of $n_{i}$ and $o w_{j}$ in all their co-occurrent messages. Then, given a message, if $O\left(n_{i}\right)$ is not found for $n_{i}$ through parse tree, we can calculate $S D\left(n_{i}, o w_{j}\right)$ as is shown in Equation 1 and finally obtain a $O\left(n_{i}\right)$ :

$$
O\left(n_{i}\right)=\underset{o w_{j} \in O S\left(n_{j}\right)}{\operatorname{Argmax}} S D\left(n_{i}, o w_{j}\right)
$$

\subsubsection{User-level sentiment}

User-level opinion regarding a topic can be easily obtained through aggregation of all message-level opinion records. Tan et. al ?? applies Factor Graph to predict users' opinion towards different aspects of a certain event, but they do not consider obtaining users' opinions from their shared information by applying semantic analysis technology, they also do not take topic information into considerations. In this paper, we define two counters $C_{i,+1}^{k}$ and $C_{i,-1}^{k}, i=1, \ldots, V, k=$ $1, \ldots, K$ to record the number of times that user $u_{i}$ expresses positive or negative opinions towards topic $t_{k}$ by scanning all $u_{i}$ 's message. Then $\Psi$ can be estimated as:

$$
\psi_{i,+1}^{k}=\frac{C_{i,+1}^{k}}{C_{i,+1}^{k}+C_{i,-1}^{k}}, \psi_{i,-1}^{k}=\frac{C_{i,-1}^{k}}{C_{i,+1}^{k}+C_{i,-1}^{k}}
$$

In addition, we define another two counters $C_{i, j, \text { agree }}^{k}$ and $C_{i, j, d i s a g r e e}^{k}$ to record the number of times $u_{i}$ and $u_{j}$ agree or disagree on topic $k$ by scanning all their "post-reply" messages. Then $\Omega$ can be estimated as:

$$
\begin{gathered}
\omega_{i, j, \text { agree }}^{k}=\frac{C_{i, j, \text { agree }}^{k}}{C_{i, j, \text { agree }}^{k}+C_{i, j, \text { disagree }}^{k}}, \\
\omega_{i, j, \text { disagree }}^{k}=\frac{C_{i, j, \text { disagree }}^{k}}{C_{i, j, \text { agree }}^{k}+C_{i, j, \text { disagree }}^{k}}
\end{gathered}
$$

The strength of tie is also important to determine the opinion influence from neighbors, regardless of positive or negative influence. Especially, for $u_{i} \in O N\left(u_{j}, t_{k}\right)$, we calculate the strength of relation by:

$$
\begin{aligned}
& s_{i, j, \text { agree }}^{k}=\frac{C_{i, j, \text { agree }}^{k}}{\sum_{u_{i} \in O N\left(u_{j}, t_{k}\right)} C_{i, j, \text { agree }}^{k}}, \\
& s_{i, j, \text { disagree }}^{k}=\frac{C_{i, j, \text { disagree }}^{k}}{\sum_{u_{i} \in O N\left(u_{j}, t_{k}\right)} C_{i, j, \text { disagree }}^{k}}
\end{aligned}
$$

In many cases, given a pair $u_{i}$ and $u_{j}$, though both of their opinions can be detected, their agreement could not be judged, for example, A supports object X while B supports $\mathrm{Y}$ on the same topic $\mathrm{Z}$, if $\mathrm{X}$ and $\mathrm{Y}$ are opposite, then A disagrees with B, else, A agrees with B. To solve the problem, a pair corpus $C o E$ is generated by applying Topic Models and manual annotation, which consists of 2,104 pairs of objects. If object $\mathrm{X}$ and $\mathrm{Y}$ are opposite, then $\operatorname{CoE}(X, Y)=0$, otherwise $\operatorname{CoE}(X, Y)=0$.

In many cases that $\operatorname{CoE}(X, Y)$ is not found, other information is utilized to detect the agree/disagree relationship between two users, in addition to the content of their messages. Based on [3,11], a metric Opinion Agreement Index $(O A I)$ is introduced to quantify the influence of $u_{i}$ on $u_{j}$ :

$$
\begin{aligned}
\text { OAI }\left(u_{i}, u_{j}\right) & =a \cdot \text { Influence }\left(u_{i}\right)+b \cdot \text { Tightness }\left(u_{i}, u_{j}\right) \\
& +c \cdot \text { Similarity }\left(u_{i}, u_{j}\right)
\end{aligned}
$$

where Influence $\left(u_{i}\right)$ is a function of the number of $u_{i}$ 's followers, Tightness $\left(u_{i}, u_{j}\right)$ is a function of the frequency of interactions (i.e. replying) between $u_{i}$ and $u_{j}$, and Similarity $\left(u_{i}, u_{j}\right)$ is a function of the cosine similarity between $\theta_{i, *}$ and $\theta_{j, *} . a, b$ and $c$ are assigned as $0.6,0.3$ and 0.1 based on empirical knowledge, respectively. $O A I\left(u_{i}, u_{j}\right)$ is used to approximate the probability that $u_{j}$ agrees with $u_{i}$ when messages' content information is not enough to detect their agreement relationship.

\subsection{Gibbs Sampling}

We use the gibbs sampling to estimate $\Theta$ and $\Phi$, with two prior hyperparameters $\alpha$ and $\beta$, respectively. Assuming that $u_{i}$ posted a message and $u_{j}$ replied to $u_{i}$ by adding a comment. If the $l$ th noun found in $u_{i}$ 's message is $n_{h}$, we sampled a topic for $u_{i}$ based on Equation 7 .

$$
\begin{aligned}
& P\left(z^{l}=t_{k} \mid x=u_{i}, w=n_{h}, \mathbf{z}^{-1}\right) \propto \\
& \frac{C_{x z}^{-l}+\alpha}{\sum_{z \in T} C_{x z}^{-l}+K \alpha} \frac{C_{z w}^{-l}+\beta}{\sum_{w \in W_{N}} C_{z w}^{-l}+N \beta}
\end{aligned}
$$

where $z^{l}=t_{k}$ denotes the assignment of the $l$ th noun in to topic $t_{k}$ and $\mathbf{z}^{-1}$ denotes all topic assignments not including $n_{h} . \quad C_{x z}^{-l}$ and $C_{z w}^{-l}$ denote the number of times topic $z$ is assigned to user $x$, and noun $w$ is assigned to topic $z$ respectively, not including the current assignment for the $l$ th noun. For user $u_{j}$, if $n_{h}$ also occurs in $u_{j}$ 's replying message, $n_{h}$ is also assigned to topic $t_{k}$ and $t_{k}$ is assigned to user $u_{j}$. For all other nouns in $u_{j}$ 's replying message, the assignment of words and topics are the executed as the same probability as shown in Equation 7. The final $\Theta$ (User-Topic distributions) and $\Phi$ (Topic-Noun distributions) can be estimated by:

$$
\theta_{x z}=\frac{C_{x z}+\alpha}{\sum_{z \in T} C_{x z}+K \alpha}, \phi_{z w}=\frac{C_{z w}+\alpha}{\sum_{w \in W_{N}} C_{z w}+N \beta}
$$

\subsection{Opinion Prediction}

Our ultimate goal is to predict a user's opinion about a topic given his/her own opinion preference and his/her neighbor's opinion. First, we need estimate four parameters $\Theta, \Phi, \Psi$ and $\Omega$. A gibbs-sampling based parameter estimation algorithm is proposed, where topic modeling, sentiment analysis and influence analysis are interwoven together. The parameters learning process goes through many iterations, and in each iteration, Gibbs sampling is used to assign nouns to topics and topics to users, and parse tree and NOAI is used to detect the opinion polarity. When the iteration is done, the four parameters are calculated. 
Based on the learning results, we would like to predict users' opinion towards some object with different topic distributions (eg, a new movie, the trend of stock price, a famous person et al.). Two factors are taken into consideration for opinion prediction. First, the historical records of topic preference and opinion distribution learned from TOIM; Second, the historical opinions of neighbors and their influence type and strength learned from TOIM. The prediction result is sampled from a sum-of-weighted probability combing the two factor together as a random process. Details are omitted due to the space limitation.

\section{EXPERIMENTATION}

\subsection{Experiment Setup}

The whole data set from Tencent Weibo is crawled from Oct 07, 2011 to Jan 05, 2012, which contains about 40,000,000 daily messages. Three objects that are popular among Tencent Weibo during the 3 months are selected: Muammar Gaddafi, The Flowers of War (chinese movie), Chinese economics, which are denoted by $O_{1}$ to $O_{3}$. The statistics are summarized in Table 1. For each object, all messages are ranked based on temporal order and the last 200 hundred are selected as testing data. Then we have total number of 1,000 messages as testing data. The rest messages are used for training.

\subsection{Prediction Performance}

Three algorithms are selected as baseline methods for comparison with TOIM: SVM (Support Vector Machine), CRF (Conditional Random Field) and JST (Joint Sentiment Topic). SVM and CRF are supervised algorithms, so we construct the training data using parse tree and opinion detection technology to auto label 5,746 messages with standard grammar structures, The attributes adopted for labelling include user name, , topic informatio, key words and their grammar related words, such as verbs, adjectives. JST is developed based on Lin's work [5], the parameters assignment is the same with TOIM. None of the above three algorithms consider the topic-level social influences as TOIM does. We also apply Map-Reduce strategy to improve the efficiency of TOIM. The precision is used to compare the prediction performance for all four algorithms. All algorithms are implemented using $\mathrm{C}++$ and all experiments are performed on a servers cluster with 36 machines, each of which contains $15 \operatorname{Intel}(\mathrm{R})$ Xeon(R) processors (2.13GHZ) and 60G memory. The data set is stored in HDFS system for Map-Reduce processing. Figure 2 shows that the precision of TOIM correlates with the number of topics. Specifically, the precision rises as the the number of topics becomes larger, with the maximum value around $75 \%$. By contrast the precisions of the other three algorithms are lower than TIOM and do not exhibit correlation with the number of topics.

\subsection{Qualitative Case Study}

TOIM can be applied to detect and analyze the public opinions around some topic. We use $O_{3}$ as a case study example. Figure 3 shows the relation between public opinion and Chinese economic. Specifically, Figure 3(a) exhibits the positive/negative opinion distribution of random selected 1,000 active users over the economics topic under O3. Obviously, many users are more concerned about development of Chinese economics, although China has achieved great eco-

Table 1: Summary of experimental data
\begin{tabular}{c|l|l|l} 
& \# of post messages & \# of reply messages & \# of users \\
\hline Total & $2,350,372$ & 959,918 & 145,327 \\
$O_{1}$ & 320,176 & 114,382 & 24,382 \\
$O_{2}$ & 591,433 & 243,876 & 31,432 \\
$O_{3}$ & 742,853 & 298,764 & 38,796
\end{tabular}

nomic success. Such concern corresponds to some important problems of Chinese economics, like extremely high house price and larger gap between rich and poor. Figure 3(b) shows that the changes of all users' positive attitude toward the topic finance market under $\mathrm{O} 3$, has a high correlation with China Hushen-300 Index (An important Financial Index in China) shown in Figure 3(c). It implies that the public opinion can reflect the real financial situation.

\section{RELATED WORK}

\subsection{Topic Model}

Since the introduction of LDA model [2], various extended LDA models have been used for topic extraction from largescale corpora. Rosen-Zvi et al. [10] introduced the AuthorTopic (AT) model, which includes author ship as a latent variable. Ramage et al. [9] introduced Labeled LDA to supervise the generation of topics via user tags. Topic models can also be utilized in sentiment analysis to correlate sentiment with topics [8].

\subsection{Sentiment Analysis and Opinion Mining}

Most of related researches mainly focused on identification of sentimental object [6], or detection of object's sentimental polarity [12] without considering the topic aspects. Mei et. al [8] and Lin et. al [5] incorporated topic models and sentiment analysis without considering the social influence. Our work attempts to integrate topic models, sentiment analysis and social influence together into a two-stage probability model.

\subsection{Social Influence Analysis}

Social influence analysis has been a hot research topic in social network research, including the existence of social influence [1], the influence maximization problem [4], and the influence at topic level [?] [7]. Those researches provide us a new perspective to investigate opinion mining from social influence perspective.

\section{CONCLUSIONS}

In this paper, we study a novel problem of social opinion influence on different topics in microblog. We proposed a Topic-level Opinion Influence Model (TOIM) to formalize this problem in a unified framework. Users' historical messages and social interaction records are leveraged by TOIM to construct their historical opinions and neighbors' opinion influence through a statistical learning process, which can be further utilized to predict users' future opinions towards some specific topic. Gibbs sampling method is introduced to train the model and estimate parameters. We experimented on Tencent Weibo and the results show that the proposed TIOM can effectively model social influence and topic simultaneously and clearly outperforms baseline methods for opinion prediction. 


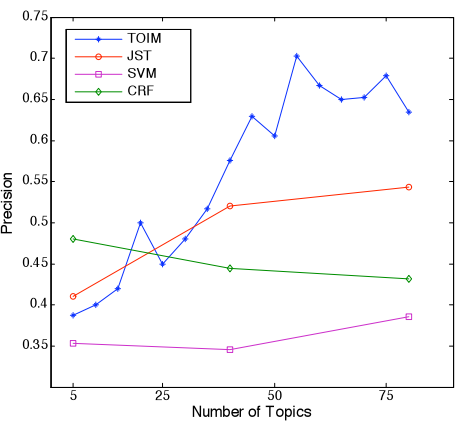

(a) $O_{1}$

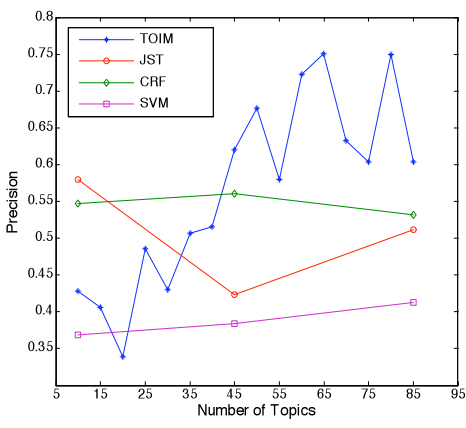

(b) $\mathrm{O}_{2}$

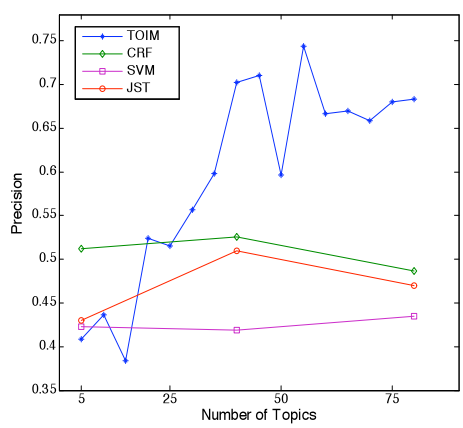

(c) $\mathrm{O}_{3}$

Figure 2: Opinion Prediction of $\mathrm{O}_{1}, \mathrm{O}_{2}, \mathrm{O}_{3}$

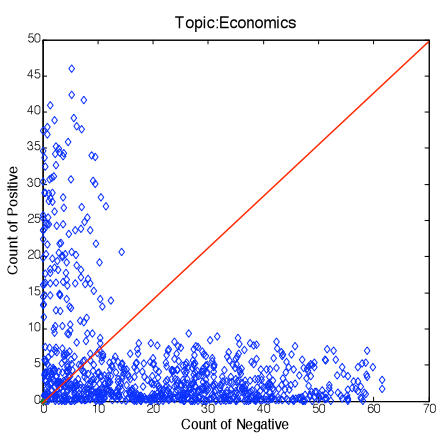

(a) opinion distribution

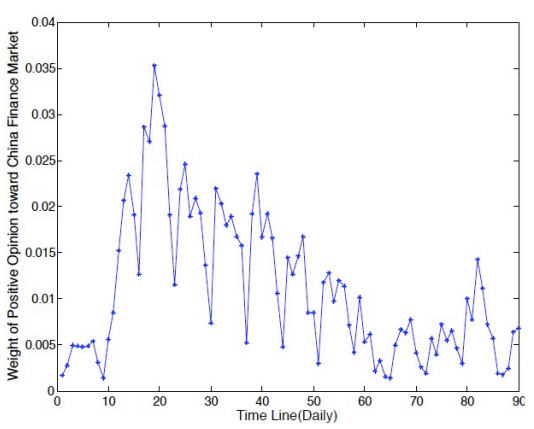

(b) positive opinion trend

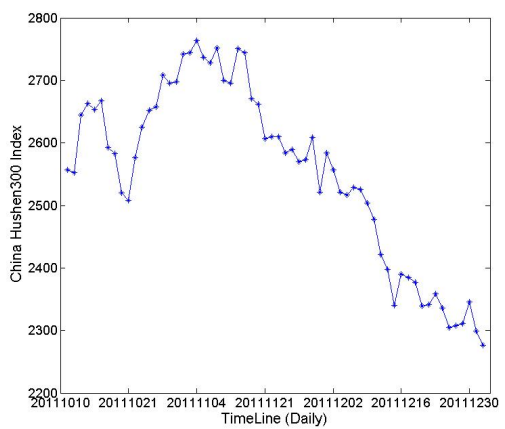

(c) financial index trend

Figure 3: Correlation between collective opinions and economic activity.

\section{ACKNOWLEDGEMENT}

This paper is supported by China Post Doc Funding (2012- M510027). National Basic Research Program of China(No.2- 011CB302302). He Gaoji Project, Tencent Company (No.2- 011ZX-01042-001-002). The National Natural Science Foun- dation of China (NSFC Program No.71072037).

\section{REFERENCES}

[1] A. Anagnostopoulos, R. Kumar, and M. Mahdian. Influence and correlation in social networks. KDD '08, pages 7-15, 2008.

[2] D. M. Blei, A. Y. Ng, and M. I. Jordan. Latent dirichlet allocation. Journal of Machine Learning Research, 3:993-1022, 2003.

[3] P. H. C. Guerra, A. Veloso, W. M. Jr., and V. Almeida. From bias to opinion: a transfer-learning approach to real-time sentiment analysis. KDD '11, pages 150-158, 2011.

[4] D. Kempe, J. Kleinberg, and E. Tardos. Maximizing the spread of influence through a social network. KDD '03, pages 137-146, 2003.

[5] C. Lin and Y. He. Joint sentiment/topic model for sentiment analysis. CIKM '09, pages 375-384, 2009.

[6] H. LIU, Y. ZHAO, B. QIN, and T. LIU. Comment target extraction and sentiment classification. Journal of Chinese Information Processing, 24:84-88, 2010.
[7] L. Liu, J. Tang, J. Han, M. Jiang, and S. Yang. Mining topic-level influence in heterogeneous networks. CIKM '10, pages 199-208, 2010.

[8] Q. Mei, X. Ling, M. Wondra, H. Su, and C. Zhai. Topic sentiment mixture: modeling facets and opinions in weblogs. WWW'07, pages 171-180, 2007.

[9] D. Ramage, D. Hall, R. Nallapati, and C. D. Manning. Labeled lda: a supervised topic model for credit attribution in multi-labeled corpora. EMNLP '09, pages 248-256, 2009.

[10] M. Rosen-Zvi, T. Griffiths, M. Steyvers, and P. Smyth. The author-topic model for authors and documents. UAI '04, pages 487-494, 2004.

[11] C. Tan, L. Lee, J. Tang, L. Jiang, M. Zhou, and P. Li. User-level sentiment analysis incorporating social networks. KDD '11, pages 1397-1405, 2011.

[12] Z. Zhai, B. Liu, H. Xu, and P. Jia. Constrained lda for grouping product features in opinion mining. PAKDD'11, pages 448-459, 2011. 